MATHEMATICS OF COMPUTATION

Volume 80, Number 275, July 2011, Pages 1813-1822

S 0025-5718(2011)02457-9

Article electronically published on January 25, 2011

\title{
UPPER BOUNDS FOR RESIDUES OF DEDEKIND ZETA FUNCTIONS AND CLASS NUMBERS OF CUBIC AND QUARTIC NUMBER FIELDS
}

\author{
STÉPHANE R. LOUBOUTIN
}

\begin{abstract}
Let $K$ be an algebraic number field. Assume that $\zeta_{K}(s) / \zeta(s)$ is entire. We give an explicit upper bound for the residue at $s=1$ of the Dedekind zeta function $\zeta_{K}(s)$ of $K$. We deduce explicit upper bounds on class numbers of cubic and quartic number fields.
\end{abstract}

\section{INTRODUCTION}

Let $K$ be an algebraic number field of degree $m=r_{1}+2 r_{2}>1$, where $r_{1}$ is the number of real places of $K$ and $r_{2}$ is the number of complex places of $K$. Let $\kappa_{K}$ be the residue at $s=1$ of the Dedekind zeta function function $\zeta_{K}(s)$ of $K$. Let $d_{K}$ be the absolute value of the discriminant of $K$. Let $h_{K}$ be its class number. Then (see [Lan, Chapter XIII, Section 3, Theorem 2]):

$$
h_{K}=\frac{w_{K} \sqrt{d_{K}}}{2^{r_{1}}(2 \pi)^{r_{2}} \operatorname{Reg}_{K}} \kappa_{K},
$$

where $w_{K} \geq 2$ is the number of complex roots of unity in $K$ and $\operatorname{Reg}_{K}$ is the regulator of $K$. To get upper bounds on $h_{K}$ we need lower bounds on $\operatorname{Reg}_{K}$ (e.g., see [Sil]) and upper bounds on $\kappa_{K}$ (e.g., see [Lou00]). If $K$ is a real quadratic number field, then

$$
h_{K} \leq \frac{1}{2} \sqrt{d_{K}}
$$

([Le] and [Ram, Corollary 2]); if $K$ is a real cyclic cubic number field, then

$$
h_{K} \leq \frac{2}{3} \sqrt{d_{K}}
$$

(see $[\mathrm{MP}$, and use [Lou93 instead of [MP, Lemme 3.2] to obtain that this bound is valid for real cyclic cubic number fields of not necessarily prime discriminants). With $e=\exp (1)$, it is known that

$$
\kappa_{K} \leq\left(\frac{e \log d_{K}}{2(m-1)}\right)^{m-1}
$$

Received by the editor November 25, 2009 and, in revised form, June 15, 2010.

2010 Mathematics Subject Classification. Primary 11R42; Secondary 11R16, 11R29.

Key words and phrases. Dedekind zeta function, number field, class number. 
([Lou00, Theorem 1] and [Lou01, Theorem 1]). If $K$ is abelian, we have a better bound:

$$
\kappa_{K} \leq\left(\frac{\log d_{K}+m \lambda_{K}}{2(m-1)}\right)^{m-1},
$$

where $\lambda_{K}=0$ if $K$ is real and $\lambda_{K}=5 / 2-\log 6$ if $K$ is imaginary (use Ram, Corollary 1] and notice that if $K$ is imaginary, then $m / 2$ of the $m$ characters in the group of primitive Dirichlet characters associated with $K$ are odd). For some totally real number fields, an improvement on (4) is known (see Lou01, Theorem $2]$ ): if $K$ ranges over a family of totally real number fields of a given degree $m>1$ for which $\zeta_{K}(s) / \zeta(s)$ is entire, there exists $C_{m}$ (computable) such that $d_{K} \geq C_{m}$ implies

$$
\kappa_{K} \leq \frac{\log ^{m-1} d_{K}}{2^{m-1}(m-1) !} \leq \frac{1}{\sqrt{2 \pi(m-1)}}\left(\frac{e \log d_{K}}{2(m-1)}\right)^{m-1} .
$$

It is known that $\zeta_{K}(s) / \zeta(s)$ is entire if $K$ is normal (see [MM, Chapter 2, Theorem $3]$ ), or if the Galois group of its normal closure is solvable (see [Uch, $\mathrm{vdW}$ ] and [MM, Chapter 2, Corollary 4.2]), e.g., for any cubic or quartic number field. This paper generalizes (6) to not necessarily totally real number fields:

Theorem 1. Let $r_{1}$ and $r_{2}$ be given, with $r_{1}+2 r_{2} \geq 3$. There exists $d_{r_{1}, r_{2}}$ effectively computable such that for any number field $K$ of degree $m=r_{1}+2 r_{2}$ with $r_{1}$ real places and $r_{2}$ complex places, we have

$$
\kappa_{K} \leq \frac{\log ^{m-1} d_{K}}{2^{m-1}(m-1) !},
$$

provided that (i) $d_{K} \geq d_{r_{1}, r_{2}}$ and (ii) that $\zeta_{K}(s) / \zeta(s)$ is entire.

For given $r_{1}$ and $r_{2}$, we will explain how to use any mathematical software, we use Maple, to compute such a $d_{r_{1}, r_{2}}$. It appears that for the small values of $r_{1}+2 r_{2}=m$, say for $3 \leq m \leq 6$, this bound (7) holds true with no restriction on the size of $d_{K}$ (in fact, we have an even better bound, see Theorem 3), the reason being that these computed $d_{r_{1}, r_{2}}$ 's are less than or equal to the least discriminants of number fields of degree $m=r_{1}+2 r_{2} \leq 6$ with $r_{1}$ real places and $r_{2}$ complex places. However, even in the simplest situation where we assume that $K$ is totally real, we could not in [Lou05] obtain beforehand a $C>0$ such that (7) holds true for $K$ 's of root-discriminants $\rho_{K}=d_{K}^{1 / m}$ greater than $C$.

Set

$$
\gamma=\lim _{m \rightarrow \infty}\left(\sum_{k=1}^{m} \frac{1}{k}-\log m\right)=0.57721 \cdots
$$

(Euler's constant) and

$$
\lambda_{r_{2}, m}=2+r_{2} \log 4-(m-1)(\log (4 \pi)-\gamma) .
$$

Since $\lambda_{r_{2}, m}<0$ for $m \geq 3$, Theorem 1 follows from the bound

$$
\kappa_{K} \leq \frac{\left(\log d_{K}+\lambda_{r_{2}, m}\right)^{m-1}}{2^{m-1}(m-1) !}+O_{r_{2}, m}\left(\log ^{m-3} d_{K}\right),
$$


where the implied constants are effective and depend on $r_{2}$ and $m$ only. To prove (8), we generalize the method introduced in [Lou96]. Set

$$
\gamma(1)=\lim _{m \rightarrow \infty}\left(\sum_{k=1}^{m} \frac{\log k}{k}-\frac{1}{2} \log ^{2} m\right)=-0.07281 \cdots
$$

and

$$
\mu_{r_{2}, m}=3+r_{2} \pi^{2} / 12-(m-1)\left(\pi^{2} / 8-\gamma^{2}-2 \gamma(1)\right) .
$$

The error term in (8) is less than or equal to zero if $\mu_{r_{2}, m}>0$ and $d_{K}$ is large enough. Now, $\mu_{r_{2}, m}>0$ if and only if we are in one of the following cases:

\section{TABLE 1}

\begin{tabular}{|rrllr|}
\hline$m$ & $r_{2}$ & $\lambda_{r_{2}, m}$ & $\mu_{r_{2}, m}$ & $d_{K} \geq$ \\
\hline 2 & 0 & $2+\gamma-\log (4 \pi)=0.04619 \cdots$ & $1.95384 \cdots$ & 3 \\
2 & 1 & $2+\gamma-\log \pi=1.43248 \cdots$ & $2.77631 \cdots$ & 3 \\
\hline 3 & 0 & $2+2 \gamma-2 \log (4 \pi)=-1.90761 \cdots$ & $0.90769 \cdots$ & 146 \\
3 & 1 & $2+2 \gamma-2 \log (2 \pi)=-0.52132 \cdots$ & $1.73015 \cdots$ & 4 \\
\hline 4 & 1 & $2+3 \gamma-\log \left(16 \pi^{3}\right)=-2.47513 \cdots$ & $0.68400 \cdots$ & 75100 \\
4 & 2 & $2+3 \gamma-\log \left(4 \pi^{3}\right)=-1.08883 \cdots$ & $1.50647 \cdots$ & 35 \\
\hline 5 & 2 & $2+4 \gamma-4 \log (2 \pi)=-3.04264 \cdots$ & $0.46031 \cdots$ & $21 \cdot 10^{10}$ \\
\hline 6 & 3 & $2+5 \gamma-\log \left(16 \pi^{5}\right)=-3.61015 \cdots$ & $0.23662 \cdots$ & $21 \cdot 10^{31}$ \\
\hline
\end{tabular}

It will follow that we have a pleasingly explicit bound:

Theorem 2. Assume that we are in one of the eight cases of Table 1. Then,

$$
\kappa_{K} \leq \frac{\left(\log d_{K}+\lambda_{r_{2}, m}\right)^{m-1}}{2^{m-1}(m-1) !}
$$

provided that $d_{K}$ is large enough, as given in the last column of Table 1.

The results in Lou93 and Lou96 are the case $m=2$ of Theorem 2 above. (However, in the quadratic case we have an even better bound (see $\mathrm{Ram}$ ).) Finally, by taking constants slightly less than these $\lambda_{r_{2}, m}$, we have a the fully explicit following result where we do not have any restriction on $d_{K}$ (compare with Theorem 1):

Theorem 3. Let $K$ be a number field of degree $m \in\{2,3,4,5,6\}$ for which $\zeta_{K}(s) / \zeta(s)$ is entire. Then,

$$
\kappa_{K} \leq \frac{\left(\log d_{K}+\lambda\right)^{m-1}}{2^{m-1}(m-1) !},
$$

where $\lambda$ is as in Table 2, 
TABLE 2

\begin{tabular}{|l|rrrr|}
\hline$m$ & $r_{2}=0$ & $r_{2}=1$ & $r_{2}=2$ & $r_{2}=3$ \\
\hline 2 & 0.04620 & 1.43249 & & \\
3 & -1.74865 & -0.52132 & & \\
4 & -2.94863 & -2.07896 & -1.08883 & \\
5 & -4.21779 & -3.29415 & -2.41877 & \\
6 & -5.49315 & -4.55901 & -3.64104 & -2.76490 \\
\hline
\end{tabular}

Corollary 4. If $K$ is a totally real cubic number field, then

$$
h_{K} \leq \frac{1}{2} \sqrt{d_{K}} .
$$

If $K$ is a totally real quartic number field which contains no quadratic subfield, then

$$
h_{K} \leq \frac{5 \sqrt{10}}{24} \sqrt{d_{K}}
$$

We refer to Dai for examples of number fields with very large class numbers.

\section{Proof of the bound (8)}

We adapt Lou00, Proof of Theorem 7]. Let $K$ be a number field of degree $m=r_{1}+2 r_{2}>1$. Assume that $\zeta_{K}(s) / \zeta(s)$ is entire. Set $A_{K / \mathbf{Q}}=\sqrt{d_{K} / 4^{r_{2}} \pi^{m-1}}$,

$$
\Gamma_{K / \mathbf{Q}}(s)=\Gamma^{r_{1}-1}(s / 2) \Gamma^{r_{2}}(s)=\frac{2^{r_{2}(s-1)}}{\pi^{r_{2} / 2}} \Gamma^{r_{1}+r_{2}-1}(s / 2) \Gamma^{r_{2}}((s+1) / 2)
$$

(notice that $r_{1}+r_{2}-1 \geq 0$ and $r_{2} \geq 0$ ) and

$$
F_{K / \mathbf{Q}}(s)=A_{K / \mathbf{Q}}^{s} \Gamma_{K / \mathbf{Q}}(s)\left(\zeta_{K}(s) / \zeta(s)\right) .
$$

Then, $F_{K / \mathbf{Q}}(s)$ is entire and $F_{K / \mathbf{Q}}(s)=F_{K / \mathbf{Q}}(1-s)$. Let

$$
S_{K / \mathbf{Q}}(x):=\frac{1}{2 \pi i} \int_{c-i \infty}^{c+i \infty} F_{K / \mathbf{Q}}(s) x^{-s} \mathrm{~d} s \quad(c>1 \text { and } x>0)
$$

denote the inverse Mellin transform of $F_{K / \mathbf{Q}}(s)$. Then,

$$
S_{K / \mathbf{Q}}(x)=\frac{1}{x} S_{K / \mathbf{Q}}\left(\frac{1}{x}\right)
$$

(notice that $F_{K / \mathbf{Q}}(s)$ is entire, shift the vertical line of integration $\Re(s)=c>1$ in (11) leftwards to the vertical line of integration $\Re(s)=1-c<0$, then use the functional equation $F_{K / \mathbf{Q}}(1-s)=F_{K / \mathbf{Q}}(s)$ to come back to the vertical line of integration $\Re(s)=c>1)$. For $\Re(s)>1$,

$$
F_{K / \mathbf{Q}}(s)=\int_{0}^{\infty} S_{K / \mathbf{Q}}(x) x^{s} \frac{\mathrm{d} x}{x}
$$

is the Mellin transform of $S_{K / \mathbf{Q}}(x)$. Using (12), we obtain

$$
F_{K / \mathbf{Q}}(s)=\int_{1}^{\infty} S_{K / \mathbf{Q}}(x)\left(x^{s}+x^{1-s}\right) \frac{\mathrm{d} x}{x}
$$


on the whole complex plane. Now, write $\zeta_{K}(s) / \zeta(s)=\sum_{n \geq 1} a_{K / \mathbf{Q}}(n) n^{-s}$ and $\zeta^{m-1}(s)=\sum_{n \geq 1} a_{m-1}(n) n^{-s}(\Re(s)>1)$. Then, $\left|a_{K / \mathbf{Q}}(n)\right| \leq a_{m-1}(n)$ (see [Lou01, $(55)])$ and

$$
S_{K / \mathbf{Q}}(x)=\sum_{n \geq 1} a_{K / \mathbf{Q}}(n) H_{K / \mathbf{Q}}\left(n x / A_{K / \mathbf{Q}}\right)
$$

where

$$
H_{K / \mathbf{Q}}(x)=\frac{1}{2 \pi i} \int_{c-i \infty}^{c+i \infty} \Gamma_{K / \mathbf{Q}}(s) x^{-s} \mathrm{~d} s .
$$

Since $H_{K / \mathbf{Q}}(x)>0$ for $x>0$ (see [Lou01, Theorem 20]) 11), we have

$$
\left|S_{K / \mathbf{Q}}(x)\right| \leq \sum_{n \geq 1} a_{m-1}(n) H_{K / \mathbf{Q}}\left(n x / A_{K / \mathbf{Q}}\right) .
$$

Plugging this into (13), we obtain

$$
\begin{aligned}
& \frac{\sqrt{d_{K}}}{(2 \pi)^{r_{2}}} \kappa_{K}=F_{K / \mathbf{Q}}(1)=\int_{1}^{\infty} S_{K / \mathbf{Q}}(x)(1+1 / x) \mathrm{d} x \\
& \leq \sum_{n \geq 1} a_{m-1}(n) \int_{1}^{\infty} H_{K / \mathbf{Q}}\left(n x / A_{K / \mathbf{Q}}\right)(1+1 / x) \mathrm{d} x \\
& =\sum_{n \geq 1} a_{m-1}(n) \frac{1}{2 \pi i} \int_{c-i \infty}^{c+i \infty}\left(\int_{1}^{\infty}\left(n x / A_{K / \mathbf{Q}}\right)^{-s}(1+1 / x) \mathrm{d} x\right) \Gamma_{K / \mathbf{Q}}(s) \mathrm{d} s \\
& =\sum_{n \geq 1} a_{m-1}(n) \frac{1}{2 \pi i} \int_{c-i \infty}^{c+i \infty}\left(\frac{1}{s-1}+\frac{1}{s}\right) \Gamma_{K / \mathbf{Q}}(s)\left(n / A_{K / \mathbf{Q}}\right)^{-s} \mathrm{~d} s \\
& =\frac{1}{2 \pi i} \int_{c-i \infty}^{c+i \infty}\left(\frac{1}{s-1}+\frac{1}{s}\right) \Gamma_{K / \mathbf{Q}}(s) \zeta^{m-1}(s) A_{K / \mathbf{Q}}^{s} \mathrm{~d} s .
\end{aligned}
$$

Therefore, we have

$$
\kappa_{K} \leq I_{K}(s):=\frac{1}{2 \pi i} \int_{c-i \infty}^{c+i \infty} f_{K}(s) \mathrm{d} s \quad(c>1),
$$

where

$$
f_{K}(s)=\tilde{\Gamma}^{r_{2}}(s) \Lambda^{m-1}(s)\left(\frac{1}{s-1}+\frac{1}{s}\right) d_{K}^{(s-1) / 2},
$$

$\Lambda(s)=\pi^{-s / 2} \Gamma(s / 2) \zeta(s)$ and $\tilde{\Gamma}(s)=\Gamma((s+1) / 2) /(\Gamma(s / 2) / \Gamma(1 / 2))$. Recall that $\Lambda(s)$ has only two poles, both simple, at $s=1$ and $s=0$, and satisfies the functional equation $\Lambda(s)=\Lambda(1-s)$. Moreover, $1 / \Gamma(s / 2)$ is entire whereas $\Gamma((s+1) / 2)$ has a simple pole at each odd negative integer. It follows that $f_{K}(s)$ has a pole of order $m>1$ at $s=1$, a pole of order $m-r_{2}=r_{1}+r_{2} \geq 1$ at $s=0$, and a pole of order $r_{2} \geq 0$ at each negative odd integer. Now, as in [Lou01, Page 1207], in the range $\sigma_{1} \leq \sigma \leq \sigma_{2}$ and $|t| \geq 1$, we have $\tilde{\Gamma}(\sigma+i t)=O(\sqrt{|t|})$ and there exists $M \geq 0$ such that $\Lambda(\sigma+i t)=O\left(|t|^{M} e^{-\pi|t| / 4}\right)$. Hence, we are allowed to shift in (14) the

\footnotetext{
${ }^{1}$ Notice the misprints in Lou00 page 273, line 1] and Lou01, Theorem 20] where one should read

$$
\left(M_{1} \star M_{2}\right)(x)=\int_{0}^{\infty} M_{1}(x / t) M_{2}(t) \frac{\mathrm{d} t}{t} .
$$
}


vertical line of integration $\Re(s)=c>1$ leftwards to the vertical line of integration $\Re(s)=1 / 2$. We pick up one residue and obtain:

$$
\kappa_{K} \leq \operatorname{Res}_{s=1}\left(f_{K}(s)\right)+I_{K}(1 / 2)=\operatorname{Res}_{s=1}\left(f_{K}(s)\right)+O_{r_{2}, m}\left(d_{K}^{-1 / 4}\right) .
$$

The bound (8) now follows from Lemma 5 below.

\section{Computation of some Residues}

To prove Theorems 2 and 3 , we need a better approximation to $I_{K}(s)$. By shifting in (14) the vertical line of integration $\Re(s)=c>1$ leftwards to the vertical line of integration $\Re(s)=-2$, we pick up three residues and we obtain:

$$
\kappa_{K} \leq \operatorname{Res}_{s=1}\left(f_{K}(s)\right)+\operatorname{Res}_{s=0}\left(f_{K}(s)\right)+\operatorname{Res}_{s=-1}\left(f_{K}(s)\right)+I_{K}(-2),
$$

where $\operatorname{Res}_{s=1}\left(f_{K}(s)\right)$ is a polynomial of degree $m-1$ in $\log d_{K}$ with real coefficients, $\sqrt{d_{K}} \operatorname{Res}_{s=0}\left(f_{K}(s)\right)$ is a polynomial of degree $r_{1}+r_{2}-1$ in $\log d_{K}$ with real coefficients, and $d_{K} \operatorname{Res}_{s=-1}\left(f_{K}(s)\right)$ is a polynomial of degree $r_{2}-1$ in $\log d_{K}$ with real coefficients. This section is devoted to computing these residues.

Lemma 5. Set

$$
f_{k, l}(s)=\tilde{\Gamma}^{k}(s) \Lambda^{l}(s)\left(\frac{1}{s-1}+\frac{1}{s}\right) e^{(s-1) X} .
$$

Then, $\operatorname{Res}_{s=1}\left(f_{k, l}(s)\right)$ is a polynomial of degree $l$ in $X$ with real coefficients and

$$
\begin{aligned}
& \operatorname{Res}_{s=1}\left(f_{k, l}(s)\right)=\frac{\left(X+A_{k, l}\right)^{l}}{l !} \quad(l=1), \\
& \operatorname{Res}_{s=1}\left(f_{k, l}(s)\right)=\frac{\left(X+A_{k, l}\right)^{l}}{l !}-C_{k, l} \frac{X^{l-2}}{(l-2) !} \quad(l=2)
\end{aligned}
$$

and

$$
\operatorname{Res}_{s=1}\left(f_{k, l}(s)\right)=\frac{\left(X+A_{k, l}\right)^{l}}{l !}-C_{k, l} \frac{X^{l-2}}{(l-2) !}+O_{k, l}\left(X^{l-3}\right) \quad(l \geq 3),
$$

where

$$
A_{k, l}=(2+k \log 4-l(\log (4 \pi)-\gamma)) / 2
$$

and

$$
C_{k, l}=\left(3+k \pi^{2} / 12-l\left(\pi^{2} / 8-\gamma^{2}-2 \gamma(1)\right)\right) / 2 .
$$

Proof. We have $\tilde{\Gamma}(s)=1+a(s-1)+b(s-1)^{2}+O\left((s-1)^{3}\right)$, with $a=\log 2$ and $b=\left(\log ^{2} 2-\pi^{2} / 12\right) / 2$, and $(s-1) \Lambda(s)=1+c(s-1)+d(s-1)^{2}+O\left((s-1)^{3}\right)$, with

$$
c=-\frac{\log (4 \pi)-\gamma}{2} \text { and } d=\frac{2(\log (4 \pi)-\gamma)^{2}+\pi^{2}-8 \gamma^{2}-16 \gamma(1)}{16} .
$$

For $k \geq 0$ and $l \geq 0$, it holds that

$$
\begin{aligned}
& \left(1+a z+b z^{2}+O\left(z^{3}\right)\right)^{k}\left(1+c z+d z^{2}+O\left(z^{3}\right)\right)^{l}\left(1+z-z^{2}+O\left(z^{3}\right)\right) \\
& \quad=1+A_{k, l} z+B_{k, l} z^{2}+O\left(z^{3}\right),
\end{aligned}
$$

where $A_{k, l}=k a+l c+1$ and $B_{k, l}=k l a c+k\left(b+\frac{k-1}{2} a^{2}\right)+l\left(d+\frac{l-1}{2} c^{2}\right)+k a+l c-1$. 
Hence, the desired results hold true with

$$
C_{k, l}=A_{k, l}^{2} / 2-B_{k, l}=\left(k\left(a^{2}-2 b\right)+l\left(c^{2}-2 d\right)+3\right) / 2 .
$$

We have $a^{2}-2 b=\pi^{2} / 12$ and $c^{2}-2 d=\gamma^{2}+2 \gamma(1)-\pi^{2} / 8$.

Lemma 6. Set $r=l-k$, let $f_{k, l}(s)$ and $A_{k, l}$ be as in Lemma 5 , and set

$$
C_{k, l}^{\prime}=\left(3-k \pi^{2} / 12-l\left(\pi^{2} / 8-\gamma^{2}-2 \gamma(1)\right)\right) / 2 .
$$

If $r=0$ or $r=1$, then

$$
\operatorname{Res}_{s=0}\left(f_{k, l}(s)\right)=(-1)^{l}(\pi / 2)^{k} \frac{\left(X-A_{k, l}\right)^{r}}{r !} e^{-X} .
$$

If $r=2$, then

$$
\operatorname{Res}_{s=0}\left(f_{k, l}(s)\right)=(-1)^{l}(\pi / 2)^{k}\left(\frac{\left(X-A_{k, l}\right)^{r}}{r !}-C_{k, l}^{\prime} \frac{X^{r-2}}{(r-2) !}\right) e^{-X} .
$$

If $r \geq 3$, then

$$
\operatorname{Res}_{s=0}\left(f_{k, l}(s)\right)=(-1)^{l}(\pi / 2)^{k}\left(\frac{\left(X-A_{k, l}\right)^{r}}{r !}-C_{k, l}^{\prime} \frac{X^{r-2}}{(r-2) !}+O_{k, l}\left(X^{r-3}\right)\right) e^{-X} .
$$

Proof. Here, $\tilde{\Gamma}(s)=\frac{\pi s}{2}\left(1-a s+b s^{2}+O\left(s^{3}\right)\right)$, with $a=\log 2$ and $b=\left(\log ^{2} 2+\right.$ $\left.\pi^{2} / 12\right) / 2$, and $s \Lambda(s)=-\left(1-c s+d s^{2}+O\left(s^{3}\right)\right)$, with $c$ and $d$ as in (17).

Lemma 7. Let $f_{k, l}(s)$ be as in Lemma 5. We have

$$
\operatorname{Res}_{s=-1}\left(f_{1, l}(s)\right)=\frac{3}{2}\left(\frac{\pi}{6}\right)^{l} e^{-2 X} .
$$

Lemma 8. It holds that

$$
\left|I_{K}(-2)\right| \leq \frac{5}{4 \pi^{2}} \frac{\Gamma\left(r_{2} / 2+1\right)}{3^{m}}\left(\frac{14}{m-1}\right)^{r_{2} / 2+1} d_{K}^{-3 / 2} .
$$

Proof. Using

$$
|\tilde{\Gamma}(-2+i t)|=\left(\frac{4+t^{2}}{1+t^{2}} \frac{\pi t}{2} \tanh \left(\frac{\pi t}{2}\right)\right)^{1 / 2} \leq \sqrt{2 \pi|t|}
$$

and

$$
|\Lambda(-2+i t)|=|\Lambda(3-i t)| \leq \frac{\zeta(3)}{\pi^{3 / 2}}|\Gamma((3-i t) / 2)|=\frac{\zeta(3)}{2 \pi} \sqrt{\frac{1+t^{2}}{\cosh (\pi t / 2)}} \leq \frac{1}{3} e^{-\pi|t| / 7},
$$

we obtain:

$$
\begin{aligned}
d_{K}^{3 / 2}\left|I_{K}(-2)\right| & \leq \frac{5}{6 \pi} \int_{0}^{\infty}|\tilde{\Gamma}(-2+i t)|^{r_{2}}|\Lambda(-2+i t)|^{m-1} \mathrm{~d} t \\
& \leq \frac{5}{2 \pi 3^{m}} \int_{0}^{\infty}(2 \pi t)^{r_{2} / 2} e^{-\pi(m-1) t / 7} \mathrm{~d} t
\end{aligned}
$$

and the desired bound. 
TABLE 3. Minimal discriminants

\begin{tabular}{|l|rrrr|}
\hline$m$ & $r_{2}=0$ & $r_{2}=1$ & $r_{2}=2$ & $r_{2}=3$ \\
\hline 2 & 5 & 3 & & \\
3 & 49 & 23 & & \\
4 & 725 & 275 & 117 & \\
5 & 14641 & 4511 & 1609 & \\
6 & 300125 & 92779 & 28037 & 9747 \\
\hline
\end{tabular}

\section{Proof of Theorems 2 and 3, and contents of Tables 1 and 2}

We use (16), the previous lemmas and Table 3 above (see Odl]).

1. If $K$ is a real quadratic field, then

$$
\kappa_{K} \leq \frac{\log d_{K}+2+\gamma-\log (4 \pi)}{2}-\frac{\log d_{K}-(2+\gamma-\log (4 \pi))}{2 \sqrt{d_{K}}}+\frac{35}{18 \pi^{2} d_{K}^{3 / 2}}
$$

is less than or equal to $\left(\log d_{K}+2+\gamma-\log (4 \pi)\right) / 2$ for $d_{K} \geq 3$.

2. If $K$ is an imaginary quadratic field, then

$$
\kappa_{K} \leq \frac{\log d_{K}+2+\gamma-\log \pi}{2}-\frac{\pi}{2 \sqrt{d_{K}}}+\frac{\pi}{4 d_{K}}+\frac{35 \sqrt{14 \pi}}{36 \pi^{2} d_{K}^{3 / 2}}
$$

is less than or equal to $\left(\log d_{K}+2+\gamma-\log \pi\right) / 2$ for $d_{K} \geq 3$.

3. If $K$ is a totally real cubic number field, then

$$
\begin{aligned}
\kappa_{K} \leq & \frac{\left(\log d_{K}+2+2 \gamma-2 \log (4 \pi)\right)^{2}}{8}-\left(3 / 2+\gamma^{2}+2 \gamma(1)-\pi^{2} / 8\right) \\
& +\frac{\left(\log d_{K}-(2+2 \gamma-2 \log (4 \pi))\right)^{2}}{8 \sqrt{d_{K}}}-\frac{3 / 2+\gamma^{2}+2 \gamma(1)-\pi^{2} / 8}{\sqrt{d_{K}}}+\frac{35}{108 \pi^{2} d_{K}^{3 / 2}}
\end{aligned}
$$

is less than or equal to $\left(\log d_{K}+2+2 \gamma-2 \log (4 \pi)\right)^{2} / 8$ for $d_{K} \geq 146$, and less than or equal to $\left(\log d_{K}-1.74865\right)^{2} / 8$ for $d_{K} \geq 49$.

4. If $K$ is a not totally real cubic number field, then

$$
\begin{aligned}
\kappa_{K} \leq & \frac{\left(\log d_{K}+2+2 \gamma-2 \log (2 \pi)\right)^{2}}{8}-\left(3 / 2+\gamma^{2}+2 \gamma(1)-\pi^{2} / 12\right) \\
& +\frac{\pi}{4 \sqrt{d_{K}}}\left(\log d_{K}-2-2 \gamma+2 \log (2 \pi)\right)+\frac{\pi^{2}}{24 d_{K}}+\frac{35 \sqrt{7 \pi}}{216 \pi^{2} d_{K}^{3 / 2}}
\end{aligned}
$$

is less than or equal to $\left(\log d_{K}+2+2 \gamma-2 \log (2 \pi)\right)^{2} / 8$ for $d_{K} \geq 4$. 
5. The other cases are easily dealt with by using any software for symbolic computation, e.g., Maple, to compute the residues which appear in (16).

\section{Proof of Corollary 4}

1. Let $K$ be a totally real cubic field. Then, $\operatorname{Reg}_{K} \geq \frac{1}{16} \log ^{2}\left(d_{K} / 4\right)$ (see Cus, Theorem 1] or [Nak, Section 2.3]). Hence,

$$
h_{K}=\frac{\sqrt{d_{K}}}{4 \operatorname{Reg}_{K}} \kappa_{K} \leq \frac{\left(\log d_{K}-1.74865\right)^{2}}{2 \log ^{2}\left(d_{K} / 4\right)} \sqrt{d_{K}} \leq \frac{1}{2} \sqrt{d_{K}} .
$$

2. Let $K$ be a totally real quartic number field which contains no real quadratic subfield. Then, $\operatorname{Reg}_{K} \geq \frac{1}{80 \sqrt{10}} \log ^{3} d_{K}$ (see [CuS, Theorem 2]). By (7) (see also [Lou01, Theorem 2, point 3]), we have $\kappa_{K} \leq \frac{1}{48} \log ^{3} d_{K}$. Hence, by (1), we obtain

$$
h_{K}=\frac{\sqrt{d_{K}}}{8 \operatorname{Reg}_{K}} \kappa_{K} \leq \frac{5 \sqrt{10}}{24} \sqrt{d_{K}} .
$$

\section{REFERENCES}

[Cus] T. W. Cusick. Lower bounds for regulators. Lecture Notes in Math., 1068, Springer, Berlin (1984), 63-73. MR756083 (85k:11052)

[Dai] R. Daileda. Non-abelian number fields with very large class numbers. Acta Arith. 125 (2006), 215-255. MR2276192(2007k:11186)

[Lan] S. Lang. Algebraic number theory (Second Edition). Graduate Texts in Mathematics 110, Springer-Verlag, New York, 1994. MR:1282723 (95f:11085)

[Le] M. Le. Upper bounds for class numbers of real quadratic fields. Acta Arith. 68 (1994), 141-144. MR1305196 (95j:11101)

[Lou93] S. Louboutin. Majorations explicites de $|L(1, \chi)|$. C. R. Acad. Sci. Paris Sér. I Math. 316 (1993), 11-14. MR1198740 (93m:11084)

[Lou96] S. Louboutin. Majorations explicites de $|L(1, \chi)|$. (Suite). C. R. Acad. Sci. Paris Sér. I Math. 323 (1996), 443-446. MR1408973 (97k:11123)

[Lou00] S. Louboutin. Explicit bounds for residues of Dedekind zeta functions, values of $L$ functions at $s=1$, and relative class numbers. J. Number Theory 85 (2000), 263-282. MR.1802716 (2002i:11111)

[Lou01] S. Louboutin. Explicit upper bounds for residues of Dedekind zeta functions and values of $L$-functions at $s=1$, and explicit lower bounds for relative class numbers of CM-fields. Canad. J. Math. 53 (2001), 1194-1222. MR1863848(2003d:11167)

[Lou05] S. Louboutin. Explicit upper bounds for the residues at $s=1$ of the Dedekind zeta functions of some totally real number fields. Séminaires \& Congrès 11 (2005), 171-178; (AGCT 2003) SMF.

[MM] R. M. Murty and V. K. Murty. Non-vanishing of L-functions and applications. Progress in Mathematics 157. Birkhäuser-Verlag, Basel, 1997. MR.1482805 (98h:11106)

[MP] C. Moser and J. J. Payan. Majoration du nombre de classes d'un corps cubique cyclique de conducteur premier. J. Math. Soc. Japan 33 (1981), 701-706. MR630633 (83a:12006)

[Nak] K. Nakamula. Certain quartic fields with small regulators. J. Number Theory 57 (1996), 1-21. MR1378570 (97h:11128)

[Odl] A. M. Odlyzko. Bounds for discriminants and related estimates for class numbers, regulators and zeros of zeta functions: a survey of recent results. Sém Théor. Nombres Bordeaux (2) 2 (1990), 119-141. MR1061762 (91i:11154)

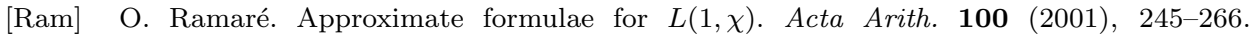
MR.1865385 (2002k:11144)

[Sil] J. H. Silverman. An inequality relating the regulator and discriminant of a number field. J. Number Theory 19 (1984), 437-442. MR769793 (86c:11094) 
[Uch] K. Uchida. On Artin L-functions. Tohoku Math. J. 27 (1975), 75-81. MR0369323 $(51: 5558)$

[vdW] R. W. van der Waall. On a conjecture of Dedekind on zeta functions. Indag. Math. 37 (1975), 83-86. MR0379439(52:344)

Institut de Mathématiques de Luminy, UMR 6206, 163, avenue de Luminy, Case 907, 13288 Marseille Cedex 9, France

E-mail address: loubouti@iml.univ-mrs.fr 